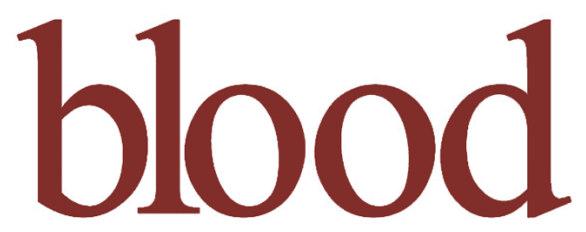

2001 97: 3513-3520

doi:10.1182/blood.V97.11.3513

\title{
Molecular characterization and expression of a novel human leukocyte cell-surface marker homologous to mouse Ly-9
}

Miguel Angel de la Fuente, Victoria Tovar, Neus Villamor, Nuria Zapater, Pilar Pizcueta, Elias Campo, Jaime Bosch and Pablo Engel

Updated information and services can be found at:

http://bloodjournal.hematologylibrary.org/content/97/11/3513.full.html

Articles on similar topics can be found in the following Blood collections

Immunobiology (5064 articles)

Signal Transduction (1930 articles)

Information about reproducing this article in parts or in its entirety may be found online at:

http://bloodjournal.hematologylibrary.org/site/misc/rights.xhtml\#repub_requests

Information about ordering reprints may be found online at:

http://bloodjournal.hematologylibrary.org/site/misc/rights.xhtml\#reprints

Information about subscriptions and ASH membership may be found online at:

http://bloodjournal.hematologylibrary.org/site/subscriptions/index.xhtml

Blood (print ISSN 0006-4971, online ISSN 1528-0020), is published weekly by the American Society of Hematology, 2021 L St, NW, Suite 900, Washington DC 20036. Copyright 2011 by The American Society of Hematology; all rights reserved.

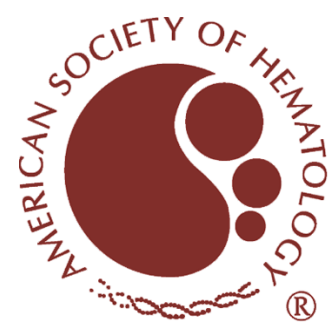




\section{Molecular characterization and expression of a novel human leukocyte cell-surface marker homologous to mouse Ly-9}

Miguel Angel de la Fuente, Victoria Tovar, Neus Villamor, Nuria Zapater, Pilar Pizcueta, Elias Campo, Jaime Bosch, and Pablo Engel

Ly-9 is a mouse cell-surface glycoprotein that is selectively expressed on thymocytes and on mature $T$ and $B$ lymphocytes. Ly-9 belongs to the CD2 subset of the immunoglobulin superfamily, an emerging family of cell signaling receptors. Recently, a partial human Ly-9 complementary DNA (cDNA) sequence has been described. Full-length cDNA clones were isolated that included the initiation codon, the sequence encoding the full signal peptide, and 14 amino acids more in the cytoplasmic domain than in the previously reported clone. The predicted extracellular domain of human Ly-9 contains 4 immunoglobulinlike domains, similar to those in mouse Ly-9. Northern blot analysis revealed that the human Ly-9 messenger RNA (2.6 kb) is expressed predominantly in lymph node, spleen, thymus, and peripheral blood leukocytes. Four monoclonal antibodies (mAbs) were raised against human $L y-9$ by immunizing mice with the pre-B-cell line 300.19 stably transfected with human Ly-9 full-length cDNA. These mAbs strongly stained the surfaces of cells transfected with human Ly-9 cDNA but not of untransfected cells. Human Ly-9 expression was restricted to $\mathrm{T}$ and $\mathrm{B}$ lymphocytes and thymocytes, with the highest levels of expression on $\mathrm{CD}^{+}{ }^{+} \mathrm{CD} 8^{-}$and $\mathrm{CD}^{-}{ }^{-} \mathrm{CD} 8^{+}$thymocytes. Monocytes, granulocytes, platelets, and red blood cells were uniformly negative for Ly-9. These mAbs immunoprecipitated major polypeptides of $\mathbf{1 2 0} \mathrm{kd}$ from the transfected cells and $\mathbf{1 2 0} \mathbf{k d}$ and $\mathbf{1 0 0}$ kd from B-cell line Daudi, probably because of the cell-surface-expressed isoforms. These data demonstrate that human Ly-9 is a new marker for the study of normal and malignant leukocytes. (Blood. 2001;97:3513-3520)

๑) 2001 by The American Society of Hematology

\section{Introduction}

Several cell-surface molecules, termed accessory molecules, cooperate with antigen-specific receptors by participating in cell adhesion between lymphocytes and other cells or by contributing to cell signal transduction. ${ }^{1}$ A significant number of these molecules belong to the immunoglobulin superfamily $(\operatorname{IgSF}){ }^{2}$

Mouse Ly-9 is a cell-surface glycoprotein expressed on the cell surfaces of thymocytes and mature B- and T-lymphocytes. It was initially described as an alloantigenic marker of lymphocyte differentiation, and it has 2 alleles. ${ }^{3,4}$ Ly-9.1 is found in most mouse strains, whereas Ly-9.2 is only found in C57BL/6 and related strains (C57BR, C57L, and C58). ${ }^{5,6}$ Because Ly-9 is broadly expressed during lymphoid differentiation, monoclonal antibodies (mAbs) against the Ly-9.1 allotype are used as markers to examine the contribution of embryonic stem-derived cells to lymphoid development in RAG-1- and RAG-2-deficient blastocyst complementation studies. ${ }^{7-9}$ These mAbs are also used to test the percentage of chimerism in allophenic mice. ${ }^{10}$

Ly-9 has also been reported to be a member of the IgSF with structural features that place it within the $\mathrm{CD} 2$ family, which includes CD48, CD58, 2B4, CD84, and CDw150. ${ }^{11,12}$ Members of this family are expressed by various leukocytes and are involved in the activation of lymphocytes and natural killer cells. ${ }^{13}$ Unlike other CD2 family members, Ly-9 has 4 rather than 2 IgSF domains in its extracellular part. Domains 1 and 3 are similar, as are domains
2 and 4, suggesting that Ly-9 arose from a progenitor with one $\mathrm{V}$ and one C2 domain. ${ }^{11}$ Ly-9 has the highest similarity to the cell-surface molecules CD84 and CD48. ${ }^{14,15}$ The Ly-9 locus lies close to the $C D 48,2 B 4, C D w 150$, and $C D 84$ genes on both mouse and human chromosome 1 . These genes may be the result of gene duplication from a common ancestor, with a second duplication event leading to the 4-domain structure of Ly-9. ${ }^{14-16}$

The function of this leukocyte-specific cell-surface molecule is unknown. However, analysis of the cytoplasmic tail of Ly-9 reveals various motifs identical to those observed in several receptors involved in cell signaling. ${ }^{17}$ The cytoplasmic tail of Ly-9 contains 2 unique tyrosine-based motifs (with the consensus amino acid sequence $\mathrm{TxYxxV/I}$ ) also found on the cytoplasmic domain of SLAM (CDw150), 2B4, and CD84. This is critical for the binding of SLAM and 2B4 to a protein called SLAM-associated protein (SAP), which is mutated in the immunodeficiency X-linked lymphoproliferative syndrome. ${ }^{18-20}$ It has been proposed that impaired signaling by these receptors is responsible for this immunodeficiency. ${ }^{13,18}$

Sandrin et $\mathrm{al}^{21}$ have reported a partial human Ly-9 complementary DNA (cDNA) sequence (HumLy-9) lacking the sequence encoding the amino-terminal portion of the signal peptide and part of the cytoplasmic tail. To date, human Ly-9 has not been serologically, biochemically, or functionally characterized. We
From the Immunology Unit, Department of Cellular Biology and Pathology, University of Barcelona Medical School; Liver Unit, Hematopathology Section, Laboratory of Anatomic Pathology, and Department of Hematology, Hospital Clínic; and Institut d'Investigacions Biomèdiques August Pi i Sunyer, Barcelona, Spain.

Submitted August 31, 2000; accepted January 31, 2001.

Supported by grants SAF97-0136 and SAF00-0037 from the Comisión Interministerial de Ciencia y Tecnología. V.T. is a Fellow of the Programa

\section{Nacional de Formación de Personal Investigador.}

Reprints: Pablo Engel, Immunology Unit, Department of Cellular Biology and Pathology, Facultad de Medicina, C/Casanovas 143, Barcelona E 08036, Spain; e-mail: engel@medicina.ub.es.

The publication costs of this article were defrayed in part by page charge payment. Therefore, and solely to indicate this fact, this article is hereby marked "advertisement" in accordance with 18 U.S.C. section 1734.

(C) 2001 by The American Society of Hematology 
have used several probes to isolate homologous full-length human Ly-9 clones. Mice were immunized with cells transfected with the isolated cDNA to raise mouse mAbs against human Ly-9. These mAbs were used to characterize the human Ly-9 glycoprotein and its surface expression on normal and malignant cells.

\section{Materials and methods}

\section{Isolation of human and Ly-9 cDNA clones}

A cDNA library from the human Burkitt lymphoma cell line Raji in $\lambda g t 11$ (Clontech Laboratories, Palo Alto, CA) was used to isolate cDNA using a mixture of various polymerase chain reaction (PCR)-generated probes corresponding to sequences reported for mouse and human surface molecule Ly9. ${ }^{11,21}$ The following primers were used to generate the probes: human primers (sense oligonucleotide 5' -CTGCTCATGGGACTAAGAG$3^{\prime}$, antisense oligonucleotide, 5'-TCTGGAGGCTCCTGGATCTGTAC$3^{\prime}$ ), corresponding to the first and second immunoglobulinlike domains ${ }^{21}$; mouse primers (sense oligonucleotide $5^{\prime}$-AGGCTCTTGCTTTAGTATTCT3', antisense oligonucleotide, 5'-ATTGCCAGATGCGGACAGGTT-3'), corresponding to the first and second immunoglobulinlike domains. ${ }^{11} \mathrm{PCR}$ probes were obtained from cDNA produced by reverse transcription (RT)-PCR of total RNA isolated from mouse peripheral lymph nodes and human peripheral blood leukocytes using a First-Strand cDNA Synthesis Kit for RT-PCR avian myeloblastis virus (AMV) and the Taq polymerase Expand High Fidelity (Boehringer Mannheim, Mannheim, Germany) according to the manufacturer's instructions. PCR products were purified, subcloned into pSP64 vector (Promega, Madison, WI), and sequenced. Random priming labeling with $\left[\alpha^{-32} \mathrm{P}\right]$ CTP was carried out using the Ready-To-Go DNA labeling kit (Pharmacia Biotech, Uppsala, Sweden). Filter hybridization was performed in ExpressHyb hybridization solution (Clontech) according to the manufacturer's instructions. Plaques that were positive on duplicate filters were picked up, and the phage insert DNA was isolated and subcloned in SP64 vector. Nucleotide sequences were determined by dideoxynucleotide chain termination using the T7 Sequencing Kit (Pharmacia Biotech). Sequence uniqueness and homology with other molecules were tested using BCM Search Launcher analysis software (Human Genome Center, Baylor College of Medicine, Houston TX). Putative leader peptides and transmembrane regions were identified using the PSORT program (Nenta Nakai, Institute for Molecular and Cellular Biology, Osaka University, Japan). The numbering system used in this paper refers to the amino acid sequence of the mature protein after cleavage of the leader peptide.

RT-PCR of human Ly-9 from transfected cells and cell lines was performed using the following oligos: 5'-GCTAGCATGAGTCAGCTGCAAATATTCTCT-3' and '5'-GCAGCAGCTGCTTTTCCTTTCAGGTGAA- ${ }^{\prime}$. PCR products were cloned using the TOPO subcloning kit (Invitrogen, Groningen, The Netherlands) and sequenced.

\section{$5^{\prime}$ rapid amplification of cDNA ends procedure}

The 5' rapid amplification of cDNA ends (RACE) was performed using the Marathon-Ready cDNA kit (Clontech Laboratories, Palo Alto, CA) according to the manufacturer's instructions. Human lymph node cDNA, which had been ligated with an adaptor, was also from Clontech. Antisense primer 5' GAACAGGGTGAATTCCTCCTCAGTGGTG-3', corresponding to the first immunoglobulinlike domain of human Ly-9, was used. PCR was carried out with an anchor primer and a nested primer corresponding to human Ly-9 sequence 5'-CCTCCTCAGTGGTGACTTCAAAATTCC-3'. One product was cloned and sequenced as described.

\section{Northern blot analysis}

Northern blot analysis was performed with nylon membranes from Clontech Laboratories (Multiple Tissue Northern Blot). Two micrograms poly $\mathrm{A}^{+}$RNA from several tissues (spleen, lymph node, thymus, appendix, peripheral leukocytes, bone marrow, fetal liver, heart, brain, placenta, lung, liver, skeletal muscle, kidney, and pancreas) was blotted after running on a denaturing formaldehyde $1.2 \%$ agarose gel. Northern blot membranes were hybridized with a 222-bp probe from immunoglobulinlike domain 1 of the human Ly9 clone and a 2000-bp human $\alpha$-actin cDNA as control probe. Hybridization was performed according to the manufacturer's instructions. Membranes were autoradiographed for 72 hours (human Ly9) or 4 hours $\left(\alpha\right.$-actin) at $-80^{\circ} \mathrm{C}$ using 2 intensifying screens.

\section{Cells}

Cell lines were cultured in RPMI 1640 media (Gibco-BRL, Gaithersburg, MD) supplemented with $10 \%$ fetal calf serum (FCS), L-glutamine, streptomycin, and penicillin. Cell lines Raji, Daudi, Jurkat, JM1, K562, HL60, RPMI, CESS, and U937 were from the American Tissue Culture Collection (Rockville, MD). The BEN cell line was obtained by the transformation of peripheral blood leukocytes with Epstein-Barr virus. Cultures of all cell lines were split the day before analysis. Blood leukocytes were isolated by Ficoll-Hypaque density gradient centrifugation of heparinized blood from healthy donors. Mononuclear cells were activated with $10 \mu \mathrm{g} / \mathrm{mL}$ phytohemagglutinin (Sigma, St Louis, MO), 5 $\mathrm{ng} / \mathrm{mL}$ and $10 \mathrm{ng} / \mathrm{mL}$ phorbol 12-myristate 13-acetate (PMA; Sigma), and $500 \mathrm{nM}$ calcium ionophore A231187 (Sigma) for 72 hours at a concentration of $2 \times 10^{6}$ cells $/ \mathrm{mL}$ in RPMI $10 \%$ FCS. Erythrocytes were isolated from the red cell pellet after Ficoll-Hypaque sedimentation of blood. Thymus and tonsil lymphocytes were isolated as described. ${ }^{22,23}$ Bone marrow cells were obtained from 3 patients with anemia. Acute leukemias were classified on the basis of morphologic, cytologic, immunologic, and cytogenetic criteria described by French-American-British and European Group for the Immunological Classification of Leukemias (EGIL) groups and the World Health Organization (WHO) ${ }^{24}$ The diagnosis of lymphoproliferative disorders was based on the Revised European-American Classification of Lymphoid Neoplasms (REAL) and WHO classification. ${ }^{25}$

All cells and tissues were obtained in accordance with protocols approved by the Ethics Committee of the Hospital Clínic of Barcelona. Informed consent was obtained from all the patients.

\section{Cell transfection}

Stably transfected cells were produced using the full-length human Ly-9 cDNA (1965 bp) cloned into the SmaI site of the pCI-neo expression vector (Promega) and the Ly-9 cDNA clone that lacked the third immunoglobulinlike domain. With the use of a Gene Pulser II Apparatus (Bio-Rad, Hercules, CA), $8 \times 10^{6} 300.19$ cells were transfected with $8 \mu \mathrm{g}$ linearized vector by electroporation $(280 \mathrm{~V}, 950 \mu \mathrm{F})$. The cells were then plated in flat-bottomed 96 -well tissue plates by limiting dilution, and stable transfectants were selected using $1.2 \mathrm{mg} / \mathrm{mL}$ G418 (Gibco-BRL). Sixteen clones from the transfection were examined by Northern blot, using a 222-bp fragment as probe. Clone 13, which expressed the highest level of message, was used in this study and was maintained in the presence of the selecting drug.

COS cells were transfected with the full-length human Ly9 cDNA subcloned in the pCI-neo expression vector and human $\mathrm{Ly} 9-\mathrm{Fc}$ chimeric constructs according to the DEAE-dextran method. ${ }^{26}$ Cell-surface expression was examined after 48 hours by immunohistochemical analysis as described. ${ }^{27}$

\section{Monoclonal antibodies}

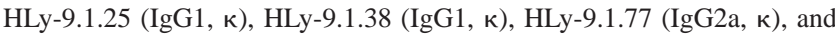
HLy-9.1.84 (IgG1, $\kappa)$ mAbs were generated by fusing NS-1 myeloma cells with spleen cells from BALB/c mice immunized twice with the mouse pre-B-cell line 300.19, stably transfected with the full-length cDNA (clone 13). Hybridomas producing $\mathrm{mAb}$ reactive to transfected but not untransfected cells were subcloned twice and used to generate concentrated supernatant obtained from the culture of the hybridomas in Integra CL 350 flasks (Integra Biosciences AG, Wallisellen, Switzerland). Monoclonal antibody isotypes were determined using the Isotyping Kit (Boehringer Mannheim). The mAbs were purified using an Affi-Gel Protein A MAPS II kit (Bio-Rad, Hercules, CA). Purified mAbs were biotinylated using Biotinamidocaproate N-hydroxysuccinimide ester (Sigma). Other mAbs 
used in this study were CD4-FITC, CD8-perCP, CD19-FITC, CD19-PE, CD19-perCP, CD38-PE, CD56-FITC, CD16-PE, and CD3-APC (Becton Dickinson, San Jose, CA). Fluorescein isothiocyanate (FITC)-conjugated antisera against human $\operatorname{IgM}$ and mouse $\operatorname{IgG}$ were from Caltag (Burlingame, CA).

\section{Immunofluorescence analysis}

Cells were kept at $4^{\circ} \mathrm{C}$ and examined immediately after isolation. Indirect immunofluorescence analysis of viable cells was carried out after the cells were washed twice (PBS containing 2\% FCS and sodium azide $0.05 \%$ ). Cells were then incubated for 20 minutes on ice, and each $\mathrm{mAb}$ was diluted to the optimal concentration for immunostaining. Isotype-matched murine $\mathrm{mAbs}$ that were unreactive with human leukocytes were used as negative controls. After they were washed, the cells were incubated for 20 minutes at $4^{\circ} \mathrm{C}$ with Avidin conjugated with FITC, phycoerythrin (PE), perCP, or Cy-Chrome (Pharmingen; Becton Dickinson). Single-, double-, triple-, and quadruple-color immunofluorescence analyses were performed on a FACSCalibur (Becton Dickinson Immunocytometry Systems, San Jose, CA). Cells with forward and light scatter properties of monocytes and granulocytes were analyzed from the blood samples. At least 5000 cells per sample were analyzed.

\section{Cell-surface biotinylation and immunoprecipitation}

Cells $\left(40 \times 10^{6}\right)$ were washed 3 times in phosphate-buffered saline (PBS), resuspended in $800 \mu \mathrm{L}$ saline, and surface-labeled with $200 \mu \mathrm{L}$ biotin 1 $\mathrm{mg} / \mathrm{mL}$ (Sigma) for 20 minutes at $4^{\circ} \mathrm{C}$. After 1 wash in RPMI media with $10 \%$ FCS and 4 washes in PBS, the cells were lysed in $1 \mathrm{~mL}$ buffer containing $0.5 \%$ (vol/ $/ \mathrm{vol}$ ) NP40 and protease inhibitors as described. ${ }^{28}$ Immunoprecipitations were carried out using $15 \mu \mathrm{g}$ mAbs. Cell lysates were precleared 3 times for 2 hours using $50 \mu \mathrm{L}(50 \% \mathrm{vol} / \mathrm{vol})$ mouse immunoglobulin-coated beads at $4^{\circ} \mathrm{C}$. Cell lysates were precleared again overnight. The precleared lysate was then incubated with $50 \mu \mathrm{L}$ CD84 $\mathrm{mAb}$-coated beads $(50 \% \mathrm{vol} / \mathrm{vol})$ or mouse immunoglobulin-coated beads with constant rotation at $4^{\circ} \mathrm{C}$ for 18 hours. Immunoprecipitates were washed and analyzed by sodium dodecyl sulfate-polyacrylamide gel electrophoresis (SDS-PAGE) as described..$^{28}$ Samples were run in the presence or absence of 5\% 2-mercaptoethanol. $\mathrm{M}_{\mathrm{r}}$ was determined using prestained standard molecular weight markers (Gibco/BRL). Proteins were transferred by electroblotting with glycine transfer buffer ( $96 \mathrm{mM}$ glycine, $12 \mathrm{mM}$ Tris base in $10 \%$ methanol, $\mathrm{pH}$ 8.3) to PVDF membranes (Immobilon; Millipore, Boston, MA). After 2 hours of incubation at room temperature with blocking solution (10\% nonfat milk in PBS), avidin peroxidase $(100 \mathrm{ng} / \mathrm{mL})$ (Sigma) was added for 1 hour. Membranes were washed with PBS with $0.1 \%$ Tween 20, and the blot was developed using enhanced chemiluminescence reagent (Amersham International, Little Chalfont, United Kingdom).

\section{Results}

Isolation and sequencing of cDNA clones encoding human Ly-9

Four different cDNA clones were isolated from a $\lambda \mathrm{gt} 11 \mathrm{cDNA}$ library constructed using RNA isolated from the human Raji cell line. Only one of these clones, measuring approximately $2100 \mathrm{bp}$ and containing an entire sequence, was selected for further analysis. This clone included the initiation codon and sequence encoding the full signal peptide and 14 more amino acids in the cytoplasmic domain than the previously reported clone. ${ }^{21}$ The sequence of the selected clone revealed an ORF encoding a 608 amino acid protein with a molecular mass of $65 \mathrm{kd}$ after cleavage of the signal peptide. The amino acid sequence was identical to mouse Ly-9 in 51\% (73\% if conserved amino acid substitutions are considered) (Figure 1), showing that it is the human homologue for this molecule. The leader peptide comprised 47 amino acids, as in mouse Ly- 9 cDNA (Figure 2). Analysis of 50 clones obtained by $5^{\prime}$
RACE from a human lymph node library showed that all presented the same leader peptide (data not shown).

The extracellular part of the molecule consisted of 4 immunoglobulinlike domains similar to those in mouse Ly-9. ${ }^{12}$ The first and third were V-like domains that lacked the usually conserved disulfide bonds between the $\beta$ sheets. The second and fourth had the structural features of an IgSF-truncated C2 set domain with 2 putative disulfide bonds. One of the isolated clones (HLy-9 no. 2) lacked the sequence corresponding to the fourth immunoglobulinlike domain (Figure 1.). The extracellular domains contained 7 putative N-linked glycosylation sites, 5 of which were preserved at the same position in mouse and human Ly-9. The human Ly-9 cytoplasmic domain was also homologous to that of mouse Ly-9 (28\% identity). The cytoplasmic domain contained 179 amino acids, 15 threonines, 16 serines, and 6 tyrosines, 5 of which were preserved in human and mouse and could be sites of phosphorylation. Tyrosines were present in 5 Tyr-containing motifs (Y-x-X-I/V) suggestive of SH2-binding sites. In contrast with results reported elsewhere, mouse and human cytoplasmic tails seemed to have the same number of amino acids (Figure 2.). Our results and previous reports differed by 14 amino acids, suggesting that these amino acids are alternatively spliced.

\section{Expression of human Ly-9 messenger RNA in several tissues}

Expression of human Ly-9 messenger RNA (mRNA) from several tissues was analyzed by Northern blot. An mRNA species of approximately $2.6 \mathrm{~kb}$ was detected in all lymphoid tissues tested, which included spleen, lymph node, thymus, appendix, peripheral leukocytes, and fetal liver, but not in bone marrow (Figure 3A-B). Slightly shorter bands, between 2.3 and $2.6 \mathrm{~kb}$, might have represented an alternatively spliced mRNA. No hybridizing mRNA was detected in the heart, brain, lung, liver, skeletal muscle, or kidney (Figure 3). Placenta was the only nonlymphoid tissue with significant levels of mRNA.

\section{Generation of mAb against human Ly-9}

Four mAbs were obtained by immunizing mice with a clone of mouse pre-B-cell line 300.19 transfected with the full-length cDNA that was shown to contain the highest amount of mRNA by Northern blot. Each mAb reacted with 300.19 and COS transfected with human Ly-9; none reacted with untransfected parenteral cells (Figure 4, and data not shown).

\section{Cell-surface staining of human cells, cell lines, and leukemias} with anti-human Ly-9 monoclonal antibodies

Anti-human Ly-9 mAbs were biotinylated and used to stain blood cells, lymphocytes isolated from thymus, bone marrow, blood and tonsil, and a large panel of lymphoid and myeloid cell lines. Anti-human Ly-9 mAb stained the cell surface of a third of the $\mathrm{CD} 4{ }^{+} \mathrm{CD} 8{ }^{+}$double-positive thymocytes (Figure 5). The intensity of Ly-9 expression was higher on $\mathrm{CD} 4^{+} \mathrm{CD} 8^{-}$and $\mathrm{CD} 4^{-} \mathrm{CD} 8^{+}$ single-positive thymocytes. Some $\mathrm{CD}^{-}{ }^{-} \mathrm{CD} 8^{-}$thymocytes expressed human Ly-9, though according to 4-color flow cytometry, most of these cells expressed CD3, whereas some staining was also seen on the $\mathrm{CD}^{-}{ }^{-} \mathrm{CD} 4{ }^{-} \mathrm{CD} 8^{-}$triple-negative cells (Figure 6). Human Ly-9 cell-surface expression correlated well with that of CD69. However, human Ly-9 appeared earlier than CD69 on the cell surfaces of the thymocytes because a significant number of Ly-9-positive thymocytes did not express CD69 (Figure 7).

Staining of peripheral blood leukocytes showed that human 


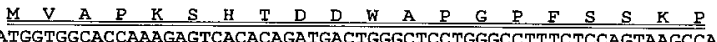

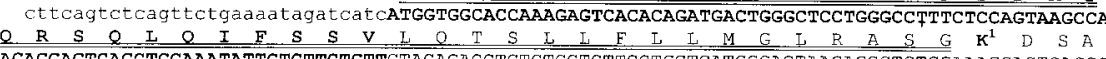

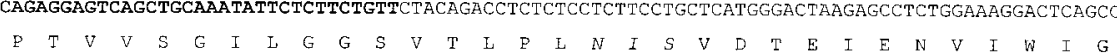
CCAACAGTGGTGTCAGGGATCCTAGGGGGTTCCGTGACTCTCCCCCTAAACATCTCAGTAGACACAGAGATTGAGAACGTCATCTGGATTGGT $\begin{array}{lllllllllllllllllllllllllllllll}\mathrm{D} & \mathrm{K} & \mathrm{N} & \mathrm{A} & \mathrm{L} & \mathrm{A} & \mathrm{F} & \mathrm{A} & \mathrm{R} & \mathrm{P} & \mathrm{K} & \mathrm{E} & N & \mathrm{~V} & \mathrm{~T} & \mathrm{I} & \mathrm{M} & \mathrm{V} & \mathrm{K} & \mathrm{S} & \mathrm{Y} & \mathrm{L} & \mathrm{G} & \mathrm{R} & \mathrm{I} & \mathrm{D} & \mathrm{I} & \mathrm{T} & \mathrm{K} & \mathrm{W} & \mathrm{S}\end{array}$ $\begin{array}{lllllllllllllllllllllllllllllllll}Y & S & \text { L } & C & I & S & N & L & T & \text { L } & \mathbb{N} & D & A & G & S & Y & K & A & Q & I & N & Q & R & \text { N } & F & \text { E } & \text { V } & \text { T } & \text { T } & \text { E } & E\end{array}$ TACTCCCTGTGCATCAGCAATCTGACTCTGAATGATGCAGGATCCTACAAAGCCCAGATAAACCAAAGGAATTTTGAAGTCACCACTGAGGAG $\begin{array}{lllllllllllllllllllllllllllllllll}E & F & T & L & F & V & Y & E & Q & L & Q & E & F & Q & V & T & M & K & S & V & K & V & S & E & N & F & C & N & I & T & L\end{array}$

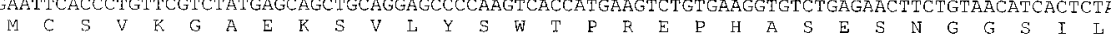
ATGTGCTCCGTGAAGGGGGCAGAGAAAAGTGTTCTGTACAGCTGGACCCCAAGGGAACCCCATGCTTCTGAGTCCARTGGAGGCTCCATTCTT

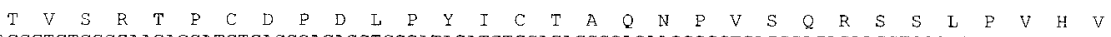
ACCGTCTCCCGAACACCATGTCACCCAGACCTGCCATACATCTGCACAGCCCAGAACCCCGTCAGCCAGAGAACCTCCCTCCCTGTCCATGT $\begin{array}{ccccccccccccccccccccccccccccccc}G & Q & F & C & T & D & P & G & A & S & R & G & G & T & T & G & E & T & V & V & G & V & L & G & E & P & V & T & L & P & L \\ \end{array}$

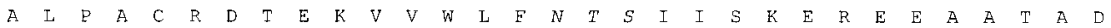
GCACTCCCAGCCTGCCGGGACACAGAGAAGGTTGTCTGGTTGTTTAACACATCCATCAT TAGCAAAGAGAGGGAAGAAGCAGCAACGGCAGAT

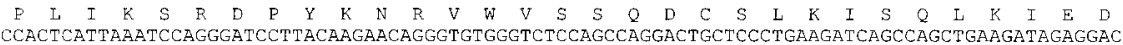

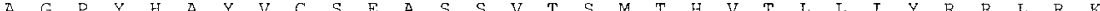
GCCGGCCCTACCATGCCTACGTGTGCTCAGAGGCCTCCAGCGTCACCAGCATGACACATGTCACCCTGCTCATCTACCGCAGGCFGAGGAAO $P \quad K \quad I \quad T$ W $S$ L $R$ H $S$ E $D$ G I C R I S I T C S V E D G G N T V $M$ CCCAAAATCACGTGGAGCCTCAGGCACAGTGAGGATGGCATCT GCAGGATCAGCCTGACCTGCTCCGTGGAGGACGGGGGAAACACTGTCATS $\begin{array}{lllllllllllllllllllllllllllllll}Y & T & W & T & P & L & Q & K & E & A & V & V & S & Q & G & E & S & H & L & N & V & S & W & R & S & S & E & N & H & P & N \\ \end{array}$ TACACATGGACCCCGCTGCAGAAGGAAGCTGTIGTGTCCCAAGGGGAATCACACCTCAATGTCTCATGGAGA.AGCAGTGAAA.ATCACCCCANO

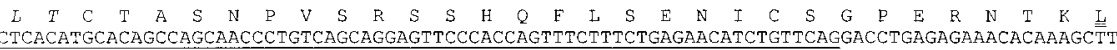
T

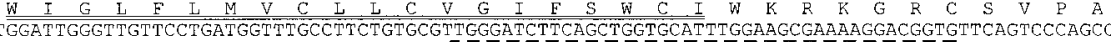
$\begin{array}{llllllllllllllllllllllllllllllll}F & C & S & S & Q & A & E & A & P & A & D & T & \text { I } & E & P & T & A & G & H & T & I & Y & S & V & I & S & G & G & Y & E & K\end{array}$ TTCTGTTCCAGCCAAGCTGAGGCCCCAGCGATACACDAGAAACCCACAGCTGGCCACACGCTATACTCTGTGCTCTCCCAAGGATAT GAGAAG $\begin{array}{lllllllllllllllllllllllllllllll}I & D & T & F & L & R & P & A & R & Q & Q & P & T & F & T & S & D & S & S & S & D & S & N & L & T & T & E & E & D & E & D\end{array}$ CTGGACACTCCCCTCAGGCCTGCCAGGCAACAGCCTACACCCACCTCAGACAGCAGCTCTGACAGCAACCTCACAACTGAGGAGGATGAGGAC $\begin{array}{lllllllllllllllllllllllllllllll}R & P & E & V & H & R & P & I & S & G & R & Y & E & V & F & D & Q & V & T & Q & E & G & A & G & H & D & P & A & P & E & G\end{array}$

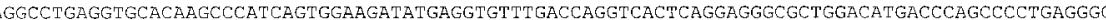
$\begin{array}{ccccccccccccccccccccccccccccccc}Q & A & D & Y & D & E & V & T & P & Y & V & T & E & V & E & S & V & V & G & E & N & T & V & Y & A & Q & V & E & N & I & Q \\ \text { CAAGCAGACTATGATCCCGTCACTCCATATGTCACGGARGT TGAGTCTGTGGTTGGAGAGACACCGTTATGCACAAGTGTTCAACTTACAG }\end{array}$

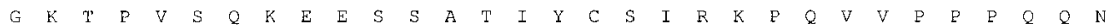
GGAAAGACCCCAGTTTCTCAGAAGGAAGAGAGCTCAGCCACAATCTACTGCTCCATACGGAPACCTCAGGTGGTGCCACCACCACAACAGAAT D L L

GATCTIGAGATTCCTGAARGTCCTACCTATGAAAATTLACCTGAaaggaaaagcagetgctgcctctetectgggaecgtggggttggaaa tcagctggacctcatggggcctggggctcgctgacagaagcacctcagaatttcettcagtgcctcagagatgcctggatgtggcccetccce

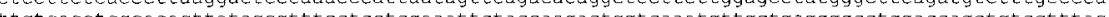

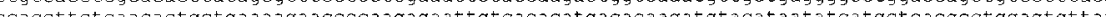

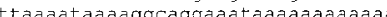

Ly-9 was expressed on mature lymphocytes but absent on monocytes, granulocytes, erythrocytes, and platelets, indicating that its expression was restricted to lymphocytes (Figure 8, and data not shown). CD $16^{+} \mathrm{CD} 56^{+}$natural killer cells expressed low amounts of this protein (Figure 8). Mature T lymphocytes expressed higher levels of human Ly-9 than B lymphocytes (Figure 8). Bone marrow CD19 B cells expressed significant amounts of human Ly-9, but expression was lower than that

\section{Leader peptide \\ LY-9 MADIKRYWCDWALGPLSENPRMSOQOIFSPILWI PLLELLMGLGASGK

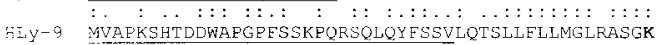

Cytoplasmic tail

Ly-9 KKKQCSSLATRYRQAEVPAELPEPETGHGQFSVLSQRYEKLDMSAKTTRHQPTPTSDTSS

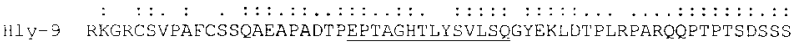

LY-9 ESSATTEEDDEKTRMHSTANSRNOLYDLVTHQDIAHALAYEGQVEYEAITPYDKVDGSMD

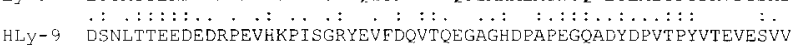

-Y-9 EEDMAYTOVSINVQGETPLPQKKEDSNTIYCSVQKPKKTAQTPQQDAESPETPTYENFT

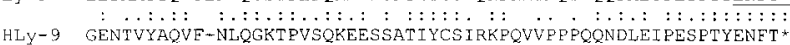

Figure 2. Amino acid comparison of leader peptide and cytoplasmic tail of mouse and human Ly-9. The upper sequence corresponds to mouse Ly-9 and the lower to human Ly-9. Identical amino acids are indicated by 2 dots; conserved substitutions are indicated by 1 dot. Gaps are indicated by a line to allow optimal alignment of the sequences. Dark lines indicate amino acids not present in previously published sequences. ${ }^{7,12}$ The GenBank accession number of the mouse cDNA sequence is AF244131.
Figure 1. Nucleotide sequence and predicted amino acid sequence of human Ly-9. Potential N-linked glycosylation sites are in italics. Signal peptide and transmembrane regions are double underlined. The asterisk indicates the termination codon. The sequence corresponding to the fourth domain is underlined. In box sequence splicing in the cytoplasmic tail. The GenBank accession number of the cDNA clone is AF244129. found on peripheral blood B lymphocytes (Figure 8). Human Ly-9 expression significantly increased on the surfaces of lymphocytes after 24-hour activation with PMA (data not shown). Three- and 4-color immunofluorescence staining of tonsil lymphocytes showed that the expression of human Ly-9 was higher on T lymphocytes than on B lymphocytes (Figure 9). $\mathrm{IgD}^{-}, \mathrm{CD} 38^{+} \mathrm{B}$ lymphocytes, corresponding to germinal center cells, expressed more human $\mathrm{Ly}-9$ than $\mathrm{IgD}^{+}, \mathrm{CD}_{3} 8^{-}$mantel zone B lymphocytes (Figure 9). Expression on the other

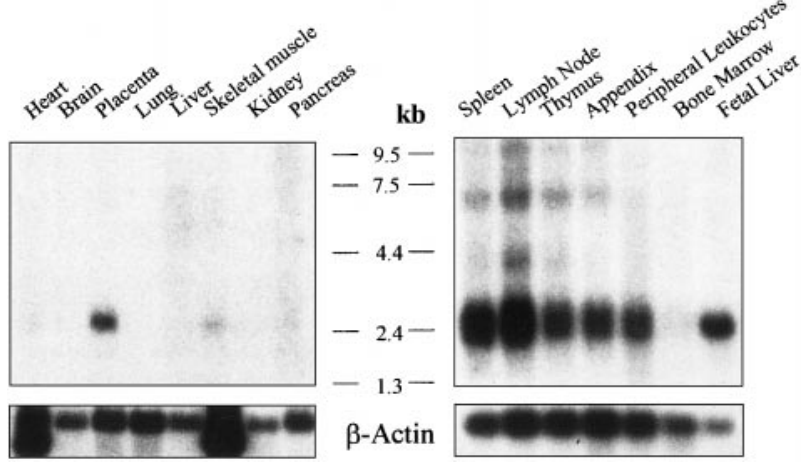

Figure 3. mRNA analysis for human Ly-9. Northern blot analysis showing the expression of human Ly-9 mRNA in lymphoid and nonlymphoid tissues. Autoradiography was scanned with a Bio-Profile densitometer (Vilber Lourmat, Marne la Vallee, France). 

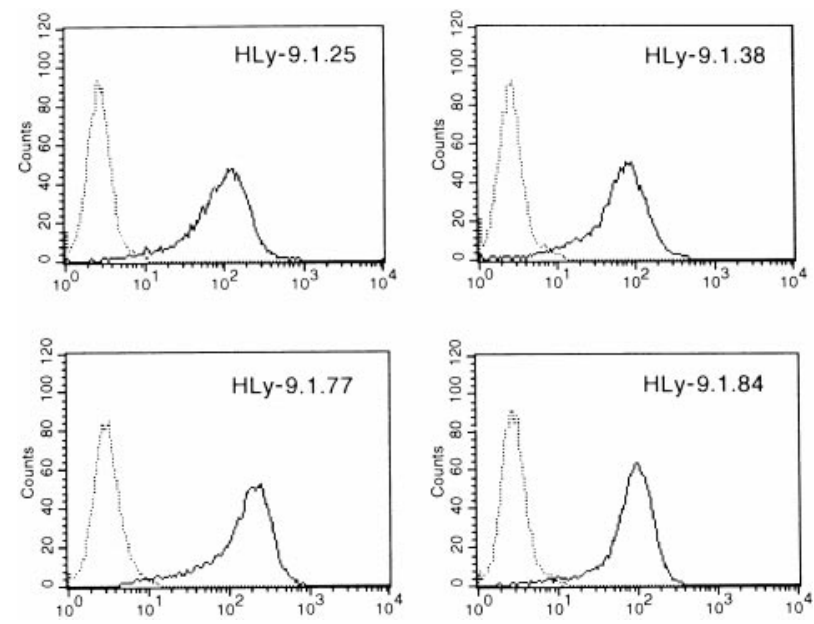

Figure 4. Monoclonal antibody reactive with the protein product of the human Ly-9 full-length cDNA. 300.19 cells transfected with HLy-9 cDNA were examined using the mAbs HLy-9.1.25, HLy-9.1.38, HLy-9.1.77, and HLy-9.1.84 in indirect immunofluorescence assays with flow cytometric analysis. Fluorescence histograms generated using 300.19 transfected cells are shown as solid lines. Staining of the 300.19 untransfected cells are shown as dotted lines. Fluorescence intensity is shown on a 3-decade log scale.

subpopulation of tonsil B cells was identical to that found on $\mathrm{IgD}^{+}, \mathrm{CD} 38^{-}$cells (data not shown).

The Burkitt lymphoma B-cell lines (Daudi and Raji) and the Epstein-Barr virus-transformed B-cell lines (CESS and BEN) expressed the highest levels of Ly-9, whereas the rest of B-cell lines expressed low levels (Table 1). Analysis of several samples of B-cell malignancies, corresponding to various stages of B-cell maturation, showed that human Ly-9 was expressed on most of them (Table 2), indicating that human Ly-9 is a pan-B-cell marker.
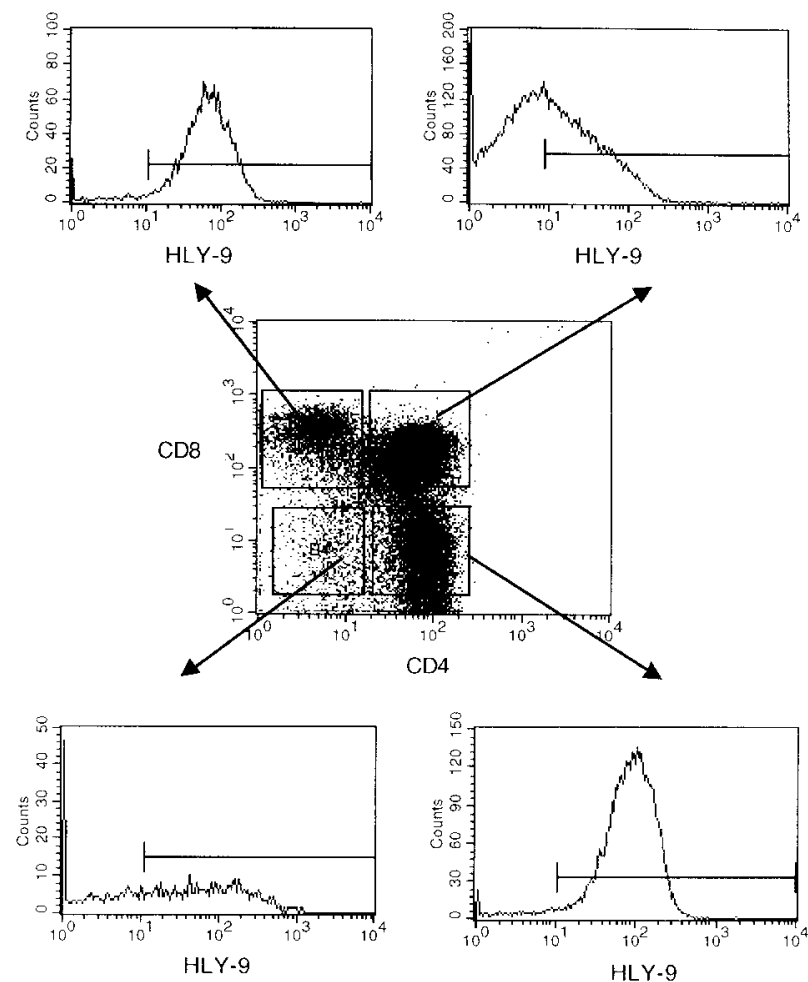

$\mathrm{HLY}-9$
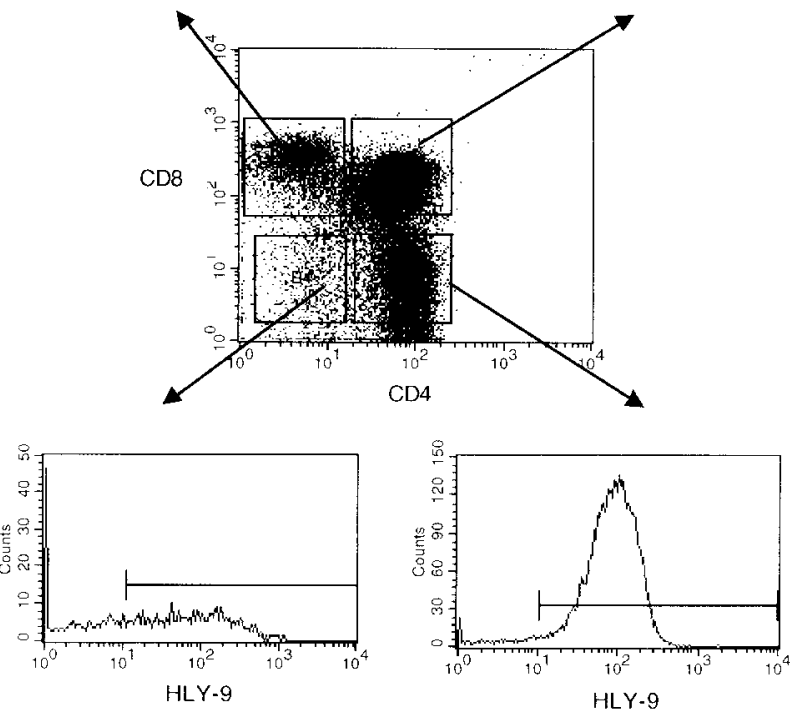

Figure 5. Ontogeny of human Ly-9 in the thymus. Flow cytometric analysis of human isolated thymocytes were incubated with biotinylated HLy-9.1.84 mAb, CD4-FITC, and CD8 perCP, then washed and incubated with avidin PE.

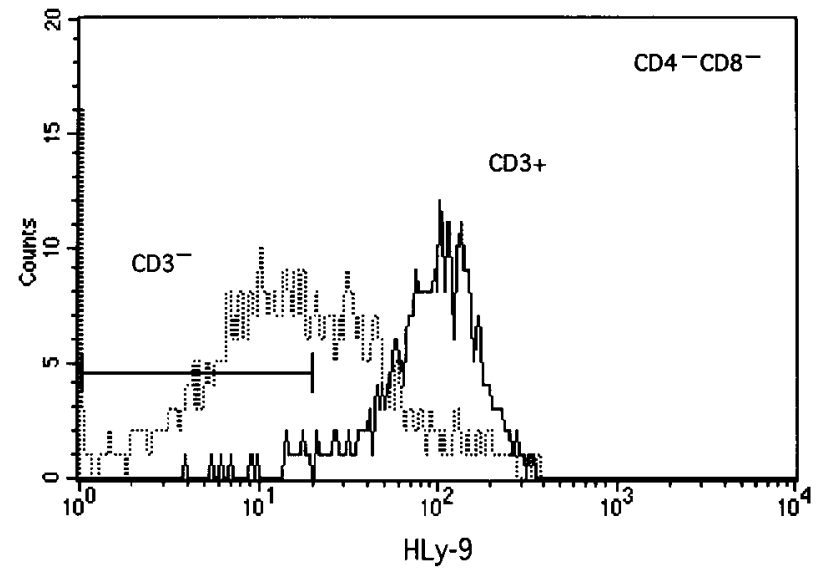

Figure 6. Four-color flow cytometric analysis of human thymocyte cell surface staining with anti-human Ly-9 mAb. Isolated thymocytes were incubated with biotinylated HLy-9.1.84 mAb, CD4-FITC, CD8 perCP, and CD3APC and then washed and incubated with avidin PE. Bar indicates the cutoff of the negative control.

T-cell line Jurkat also expressed significant amounts of human Ly-9. Myelomonocytic cell lines HL60 and U937 and erythromyeloid cell line K562 were negative for Ly-9 (Table 1). However, half the acute myeloblastic leukemias tested were positive for Ly-9 (Table 2).

\section{Immunoprecipitation of human Ly-9 from 300.19 cells transfectants and B cell-line Daudi with anti-human Ly-9 monoclonal antibodies}

We performed immunoprecipitations from biotinylated untransfected or human Ly-9 cDNA-transfected 300.19 cells using the anti-human Ly-9 mAb (Figure 10). The mAb precipitated a single band of approximately $120 \mathrm{kd}$ in reducing and nonreducing conditions. In contrast, when the same antibodies were used to precipitate human Ly-9 from the B-cell line Daudi, 2 bands of $120 \mathrm{kd}$ and $100 \mathrm{kd}$ were seen (Figure 10A). The second band was similar to that detected when 300.19 cells were transfected with the isoform that lacks the fourth immunoglobulinlike domain. To test whether the second band found in Daudi cells corresponded to an alternatively spliced form of Ly-9, RT-PCR was performed with RNA isolated from Daudi cells. Two major bands were detected (Figure 10B), and, after subcloning and

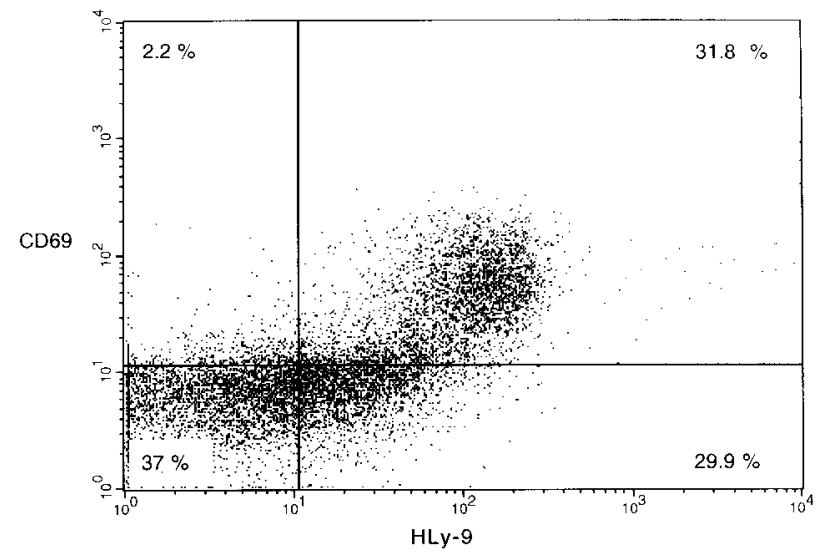

Figure 7. Two-color flow cytometric analysis of human thymocyte cell surface staining with anti-human Ly-9 mAb and CD69-FITC. Isolated thymocytes were incubated with biotinylated HLy-9.1.84 mAb and CD69-FITC washed and incubated with avidin phycoerythrin. 
From bloodjournal.hematologylibrary.org at Harvard Libraries on August 26, 2013. For personal use only.

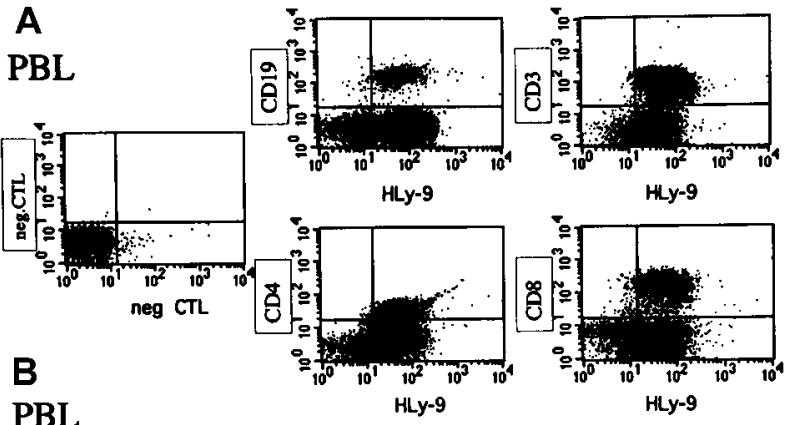

PBL
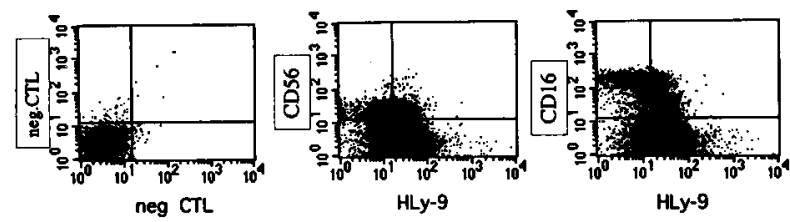

C

Bone marrow
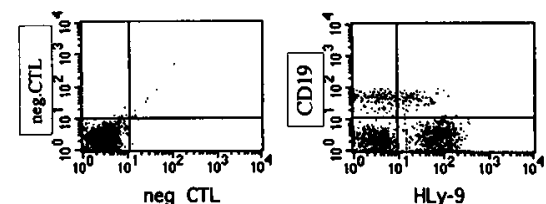

D
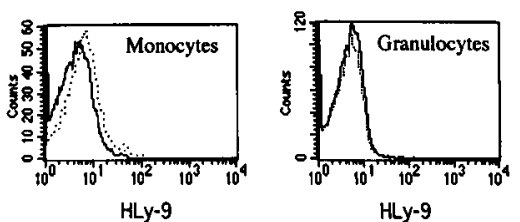

HLy-9

Figure 8. Flow cytometric analysis of human peripheral blood leukocytes and bone marrow cells staining with anti-human Ly- 9 mAb. These results are representative of those obtained in at least 6 experiments for blood samples and 3 for the bone marrow cells. PBL indicates peripheral blood leukocyte. (A) Peripheral blood mononuclear cells (PBMCs) were incubated with biotinylated HLy-9.1.84 mAb and CD19-FITC, CD3-APC, CD4-FITC, or CD8-perCP washed and incubated with avidin PE. (B) PBMCs were incubated with biotinylated HLy-9.1.84 mAb and CD56-FITC and CD16-PE washed and incubated with avidin-Cy-Chrome. (C) Bone marrow cells were stained with biotinylated HLy-9.1.84 mAb and CD19-FITC washed and incubated with avidin-PE. Cells with forward and light scatter characteristics of lymphocytes were gated for experiments shown in panels A, B, and C. (D) Periphera blood cells were stained with biotinylated HLy-9.1.84 mAb washed and incubated with avidin-PE. Cells with forward and light scatter characteristics of monocytes or granulocytes were gated. Dotted lines indicate staining of the negative control in histograms.
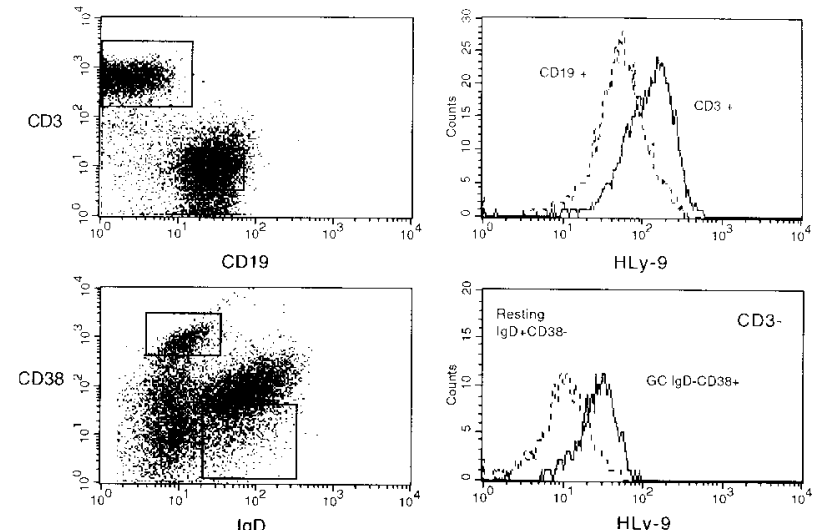

HLy-9

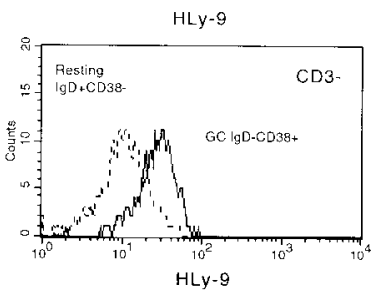

Figure 9. Flow cytometric analysis of human tonsil cell surface staining with anti-human Ly-9 mAb. Cells were incubated with biotinylated HLy-9.1.84 mAb, CD19FITC, and CD3-APC washed and incubated with avidin phycoerythrin (upper panel) and with IgD-FITC, CD38-PE, CD3-APC and incubated with avidin perCP (lower panel).
Table 1. Expression of human Ly-9 on lymphoid and myeloid cell lines

\begin{tabular}{lcc}
\hline & $\begin{array}{c}\text { Positive cells } \\
(\%)\end{array}$ & $\begin{array}{c}\text { Log mean fluorescence } \\
\text { intensity }\end{array}$ \\
\hline Raji & $\mathbf{7 6 . 4}$ & $\mathbf{3 1 . 1 0}$ \\
Daudi & 99.8 & 36.09 \\
CESS & $\mathbf{9 0 . 5}$ & $\mathbf{2 1 . 9 6}$ \\
BEN* & $\mathbf{7 6 . 3}$ & $\mathbf{1 8 . 9 5}$ \\
RPMI 8222 & $\mathbf{2 1 . 0}$ & $\mathbf{7 . 0 8}$ \\
JM1 & 13.1 & 4.75 \\
Jurkat & 76.0 & $\mathbf{1 7 . 3 7}$ \\
HSB2 & 7.5 & 4.17 \\
HL60 & 4.1 & 3.79 \\
U937 & 5.4 & 4.20 \\
K562 & 12.5 & 4.32 \\
\hline
\end{tabular}

Cells were stained with biotinylated HLy-9.1.84 mAb. This was followed by incubation with avidin-phycoerythrin and analysis by flow cytometry. An unreactive $\mathrm{mAb}$ was used as negative control. Results represent the percentage of positive cells and the mean of fluorescence intensity. Positive staining appears in boldface.

sequencing, they were shown to encode the full-length cDNA and an alternatively spliced isoform lacking the fourth immunoglobulinlike domain, respectively. The sequence of 20 independent clones of the lower band showed that they all lacked the sequence corresponding to the fourth domain. Thus, the spliced variant isolated from the cDNA might have been expressed on the surface of certain B cells.

\section{Discussion}

This paper describes the cloning and characterization of the human surface molecule Ly-9. We report the isolation of cDNA encoding full-length human Ly-9 from a human Raji cDNA library. Unlike the recently reported human Ly- 9 cDNA sequence, ${ }^{21}$ our cDNA sequence contains the complete leader sequence and part of the cytoplasmic domain. In contrast with the published sequence, our results show that mouse and human leader peptides have the same number of amino acids (Figures 1,2). Although the predicted leader peptide of both human and mouse Ly-9 is long, some other cell-surface protein, including CD49e, CD62P, CD163, and CD129, have been shown to contain long leader peptides of 40 or more amino acids.

The number of amino acids of the cytoplasmic tail is identical in

Table 2. Expression of human Ly-9 on hematopoietic malignancies

\begin{tabular}{lc} 
B-cell malignancies & \\
ALL & $2 / 2$ \\
CLL & $12 / 15$ \\
MCL & $5 / 5$ \\
HCL & $1 / 2$ \\
MM & $4 / 4$ \\
T-cell malignancies & \\
ALL & $1 / 1$ \\
Sézary & $1 / 1$ \\
Acute myeloid leukemia & \\
AML & $8 / 16$ \\
\hline
\end{tabular}

ALL indicates acute lymphocytic leukemia; CLL, chronic lymphocytic leukemia $\mathrm{MCL}$, mast cell leukemia; $\mathrm{HCL}$, hairy cell leukemia; MM, multiple myeloma; $\mathrm{AML}$, acute myeloid leukemia.

Cells were stained with biotinylated HLy-9.1.84 mAb. This was followed by incubation with avidin-phycoerythrin and analysis by flow cytometry. An unreactive mAb was used as negative control. Results represent positive samples versus analyzed samples. 


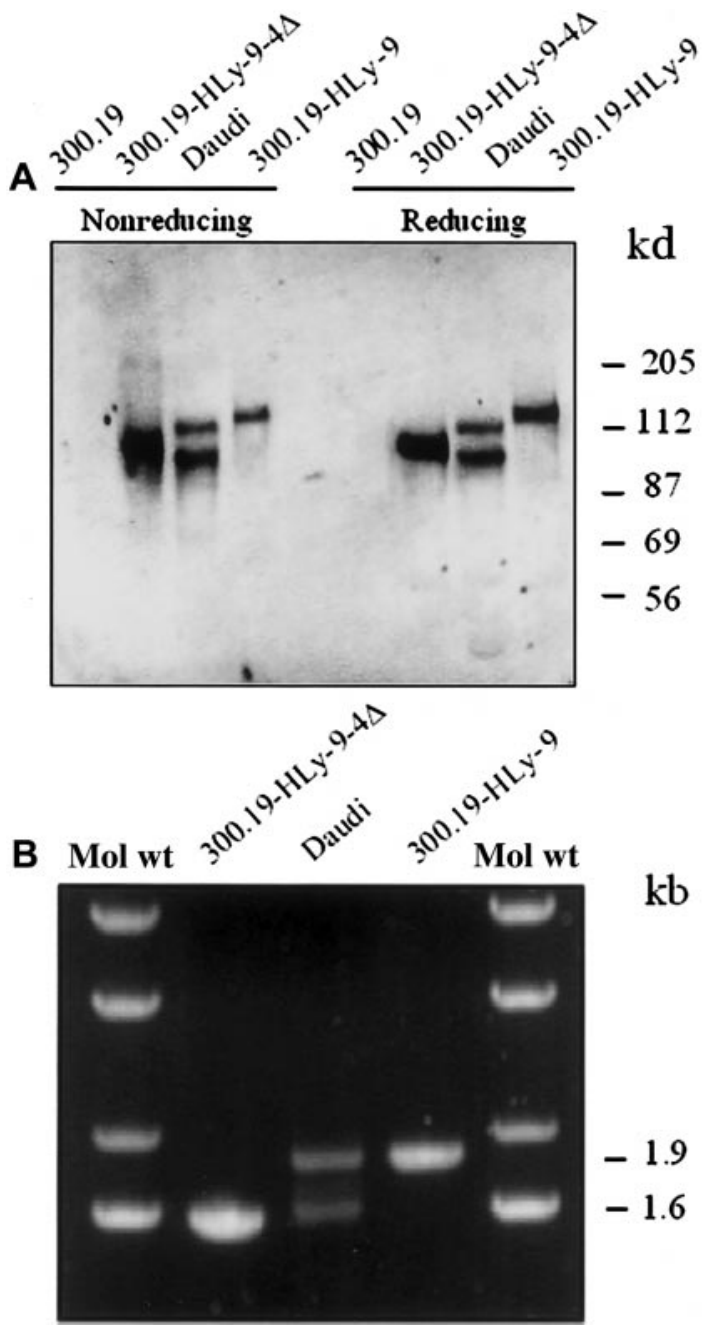

Figure 10. Immunoprecipitation and isoforms of cell surface human Ly-9 protein. (A) Detergent lysates of surface-labeled Daudi cell line, 300.19 cells transfected with full-length human Ly-9 cDNA, Ly-9 cDNA lacking the fourth immunoglobulinlike domain (HLy-9- $\Delta 4$ ), and 300.19 untransfected cells were immunoprecipitated with the HLy-9.1.25 mAb and analyzed under reducing and nonreducing conditions on a $10 \%$ sodium dodecyl sulfate-polyacrylamide gel. Molecular weights $(\mathrm{kd})$ were determined by the migration of a known protein standard. (B) cDNA was made with mRNA from 300.19 cells transfected with full-length human Ly-9 cDNA, Ly-9 cDNA lacking the fourth immunoglobulinlike domain (HLy-9- $\Delta 4)$, and B-cell line Daudi. Ethidium bromide staining of the PCR products after gel analysis. A molecular weight ladder is shown as an estimate of DNA band size.

mouse and human, except for one amino acid. ${ }^{11,29}$ The lack of 14 amino acids in the cDNA reported elsewhere may be due to mRNA splicing. ${ }^{29}$ Whether the presence of a spliced variant of the cytoplasmic tail has any biologic significance remains unclear. However, it may have, because the spliced sequence contains 2 threonines, 2 serines, and 1 tyrosine in a YxxL putative SH2binding motif (Figure 2). Two other tyrosine residues in the cytoplasmic tail of human and mouse Ly-9 are in a motif in agreement with the consensus sequence $\mathrm{T}-\Phi-\mathrm{Y}-\mathrm{X}-\mathrm{X}-\mathrm{V} / \mathrm{I}$ for the binding of SAP and SHP-2, found in SLAM and 2B4. ${ }^{18-20}$ This observation suggests that SAP and SHP-2 or other related SH2binding domain proteins may bind to Ly-9.

Northern blot analysis showed the expression of a 2.6-kb Ly-9 mRNA in lymphoid tissues. The pattern of expression is consistent with that of mouse Ly-9, which is found on mature $\mathrm{T}$ and $\mathrm{B}$ lymphocytes and thymocytes. ${ }^{3-5}$ Slightly differently sized bands between 2.3 and $2.6 \mathrm{~kb}$ were observed in most lymphoid tissues and might represent alternatively spliced mRNA encoding several isoforms of the molecule.

Four mAbs were generated by immunizing mice with a stable transfectant of human Ly-9. These mAbs stained 300.19 pre-B-cell line transfected with human Ly-9 cDNA but did not stain untransfected 300.19 cells. Using these mAbs, we showed that human Ly-9 protein was expressed on the cell surfaces of thymocytes. The expression of Ly-9 seems to have preceded positive selection during T-cell differentiation because Ly-9 appeared before CD69. CD69 is known to appear on thymocyte surfaces that have undergone positive selection. ${ }^{30}$ In addition, peripheral blood and tonsil $\mathrm{T}$ and B lymphocytes expressed significant levels of human Ly-9. The intensity of expression of human Ly-9 on B cells was lower than on $\mathrm{T}$ lymphocytes. Bone marrow B cells expressed low levels of human Ly-9, indicating that its expression increased with cell maturation. Most B-cell lines, leukemias, and lymphomas expressed human Ly-9. All multiple myeloma samples analyzed were positive for Ly-9, indicating that its expression is not lost during differentiation to plasma cells, whereas most differentiation antigens expressed on B cells were no longer expressed on terminally differentiated plasma cells. Monocytes, granulocytes, erythrocytes, and platelets did not express significant amounts of human Ly-9. Overall, these results suggest that the expression of human Ly-9 is restricted to lymphocytes. However, a significant number of acute myeloblastic leukemias expressed human Ly-9, indicating that some cells of the myeloid lineage also express human Ly-9.

The anti-human Ly-9 mAbs specifically immunoprecipitated a single polypeptide of $120 \mathrm{kd}$ from the cells transfected with full-length cDNA. However, when Ly-9 was immunoprecipitated from the B-cell line Daudi, 2 bands of $120 \mathrm{kd}$ and $100 \mathrm{kd}$ could be observed. The smaller band is probably a spliced form of the molecule lacking the fourth immunoglobulinlike domain. A band of similar size was observed when anti-human Ly-9 mAbs were used to immunoprecipitate this protein with cells transfected with the cDNA of clone HLy- $9 \Delta 4$, which lacks the fourth immunoglobulinlike domain. Minor bands are present in anti-human Ly-9 immunoprecipitates from resting peripheral blood lymphocytes and thymocytes (data not shown). These smaller bands were also observed in the initial description of mouse Ly-9 and were attributed to differences in the level of glycosylation. ${ }^{3}$ In contrast, we believe that these bands result from alternatively spliced Ly- 9 mRNAs. RT-PCR in Daudi cells showed the presence of 2 major bands. They corresponded to the full-length cDNA and a spliced variant lacking the fourth immunoglobulinlike domain of Ly-9. Nevertheless, the contribution of phosphorylation and glycosylation to the heterogeneity of bands remains to be elucidated.

Thymocytes and $\mathrm{B}$ and $\mathrm{T}$ lymphocytes express a previously undescribed glycoprotein representing the human homologue of mouse Ly-9. This protein can be used as a surface marker to study lymphocyte differentiation. The mAbs generated in this study may improve our understanding of the pattern of expression of Ly-9 and the delineation of the cytoplasmic and cell-surface molecules with which it may interact. These mAbs will be useful to establish hypotheses regarding the biologic roles of this molecule in lymphocyte development and activation.

\section{Acknowledgments}

We thank Isabel Sánchez for assistance in these experiments and Dr M. Streuli for providing 300.19 cells. 
From bloodjournal.hematologylibrary.org at Harvard Libraries on August 26, 2013. For personal use only.

\section{References}

1. Weiss A, Littman DR. Signal transduction by lymphocyte antigen receptors. Cell. 1994; 76:263274

2. Williams A-F, Barclay AN. The immunoglobulin superfamily-domains for cell surface recognition. Ann Rev Immunol. 1998;88:381-405.

3. Ledbetter JA, Golding JW, Tsu TT, Herzenberg LA. A new mouse lymphoid alloantigen (Lgp100) recognized by a monoclonal rat antibody. Immunogenetics. 1979;8:347-360.

4. Mathieson BJ, Sharrow SO, Bottomly K, Fowlkes BJ. Ly-9, an alloantigenic marker of lymphocyte differentiation. J Immunol. 1980;125:2127-2136.

5. Hogarth PM, Graig J, McKenzie I-FC. A monoclonal antibody detecting the Ly-9.2 (Lgp-100) cellmembrane alloantigen. Immunogenetics. 1980; 11:65-74.

6. Kozak CA, Davidson WF, Morse HC III. Genetic and functional relationship of the retroviral and lymphocyte alloantigen loci on mouse chromosome 1. Immunogenetics. 1984;19:163-168.

7. Arroyo AG, Yang JT, Rayburn H, Hynes RO. Differential requirements for $\alpha 4$ integrins during fetal and adult hematopoiesis. Cell. 1996;85: 997-1008.

8. Porcher C, Swat W, Rockwell K, Fujiwara Y, Alt F-W, Orkin SH. The T cell leukemia oncoprotein $\mathrm{SCL} / \mathrm{tal}-1$ essential for development of all hematopoietic lineages. Cell. 1996;86:47-57.

9. Potocnik AJ, Kohler H, Eichmann K. Hemato-lymphoid in vivo reconstitution potential of subpopulations derived from in vitro differentiated embry onic stem cells. Proc Natl Acad Sci U S A. 1997; 94:10295-10300.

10. Shechler JMG, Lawler A, Hartley JW, et al. Induction of murine acquired immunodeficiency syndrome (MAIDS) in allophenic mice generated from strains susceptible and resistant to disease. J Exp Med. 1996;184:2101-2108.

11. Sandrin MS, GumLey TP, Henning MM, et al. Isolation and characterization of cDNA clones for mouse Ly-9. J Immunol. 1992;149:1636-1641.

12. Davis SJ, van der Merwe PA. The structure and ligand interactions of CD2: implications for T-cel function. Immunol Today. 1996;617:177-187.

13. Tangye SG, Phillips JH, Lanier LL. The CD2-subset of the Ig superfamily of cell surface molecules: receptor-ligand pairs expressed by NK cells and other immune cells. Semin Immunol. 2000;12:149-157.

14. De la Fuente MA, Pizcueta P, Nadal M, Bosch J, Engel P. CD84 leukocyte antigen is a new member of the Ig superfamily. Blood. 1997;90:23982405.

15. De la Fuente MA, Tovar V, Pizcueta P, Nadal M, Bosch J, Engel P. Molecular cloning, characterization and chromosomal localization of the mouse homologue of CD84 a member of the CD2 family of cell surface molecules. Immunogenetics. 1999;49:249-255.

16. Kingsmore SF, Souryal CA, Watson M-L, Patel $\mathrm{DD}$, Seldin M-F. Physical and genetic linkage of the genes encoding Ly- 9 and CD48 on mouse and human chromosome 1. Immunogenetics. 1995;42:59-62.

17. Songyang Z, Shoelson SE, McGlade J, et al. Specific motifs recognized by $\mathrm{SH} 2$ domains of Csk, 3BP2, fps/fes, GRB-2, HCP, Syk, and Vav. Mol Cell Biol. 1994;14:2777-2785.

18. Sayos J, Wu C, Morra M, et al. The X-linked lymphoproliferative-disease gene product SAP regulates signals induced through the co-receptor SLAM. Nature. 1998;395:462-469.

19. Tangye SG, Lazetic S, Woollatt E, Sutherland GR, Lanier LL, Phillips JH. Human 2B4, an activating NK cell receptor, recruits the protein tyrosine phosphatase SHIP-2 and the adaptor signaling protein SAP. J Immunol. 1999;162:69816985.

20. Nichols KE, Harkin DO, Levitz S, et al. Inactivating mutations in a SH-2 domain-encoding gene in X-linked lymphoproliferative syndrome. Proc Natl Acad Sci U S A. 1998;95:13765-13770.

21. Sandrin MS, Henning MM, Lo MF, Baker E, Sutherland GR, McKenzie IFC. Isolation and characterization of cDNA clones for HumLy9: the human homologue of mouse Ly9. Immunogenetics 1996;43:13-19.

22. Vanhecke D, Leclercq G, Plum J, Vanderckhove $B$. Characterization of distinct stages during the differentiation of human $\mathrm{CD}_{69}{ }^{+} \mathrm{CD} 3^{+}$thymocytes and identification of thymic emigrants. J Immunol. 1995; 155:1862-1872.

23. Ingles J, Engel P, de la Calle O, Gallart T. Differential responsiveness of human $B$ lymphocytes to phorbol ester and calcium ionophore based on their state of activation. Immunology. 1989;67: 359-364.

24. Rothe G, Schitz G. Consensus protocol for the flow cytometric immunophenotyping of hematopoietic malignancies: working group on flow cytometry and image analysis. Leukemia. 1996;10: 877-895.

25. Harris NL, Jaffe ES, Stein $\mathrm{H}$, et al. A revised Eu ropean-American classification of lymphoid neoplasms: a proposal from the International Lymphoma Study Group. Blood. 1994;1361-1392.

26. Engel P, Nojima I, Rothstein D, et al. The same epitope on CD22 of B lymphocytes mediates the adhesion of erythrocytes, T and B lymphocytes, neutrophils and monocytes. J Immunol. 1993 150:4719-4732.

27. Engel, P, Wagner N, Miller A, Tedder TF. Identification of the ligand binding domains of CD22, a member of the immunoglobulin superfamily that uniquely binds a sialic acid-dependent ligand. J Exp Med. 1995;181:1581-1586.

28. Tedder TF, Schlossman SF. Phosphorylation of the B1 (CD20) cell surface molecule expressed by normal and malignant human B lymphocytes. J Biol Chem. 1988;263:10009-10015.

29. Tovar V, de la Fuente MA, Pizcueta P, Bosch J, Engel P. Gene structure of the mouse leukocyte cell-surface molecule Ly9. Immunogenetics. 2000;51:788-793.

30. Yamashita I, Nagata T, Tada T, Nakayama T. CD69 cell surface expression identifies developing thymocytes which augmentation for T cell antigen receptor-mediated positive selection. Int Immunol. 1993:5:1139-1150. 